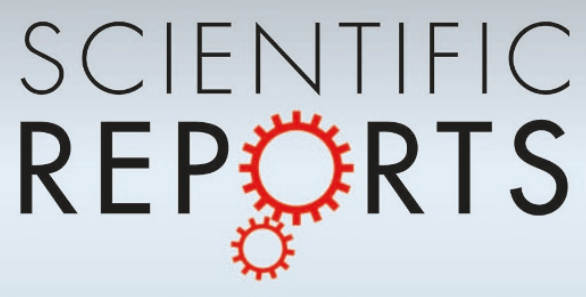

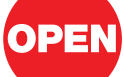

SUBJECT AREAS:

ASSAY SYSTEMS

LAB-ON-A-CHIP

HIGH-THROUGHPUT SCREENING

BIOMATERIALS-CELLS

Received

25 October 2012

Accepted

17 January 2013

Published

1 February 2013

Correspondence and requests for materials should be addressed to N.Y. (n-yosi44@agr. nagoya-u.ac.jp) or S.K. (skuroda@agr. nagoya-u.ac.jp)

\section{An automated system for high-throughput single cell-based breeding}

\author{
Nobuo Yoshimoto', ${ }^{1,}$, Akiko Kida' ${ }^{1}$ Xu Jie ${ }^{3}$, Masaya Kurokawa ${ }^{4}$, Masumi lijima' ', Tomoaki Niimi' \\ Andrés D. Maturana', Itoshi Nikaido ${ }^{5}$, Hiroki R. Ueda ${ }^{5}$, Kenji Tatematsu², Katsuyuki Tanizawa ${ }^{2}$, \\ Akihiko Kondo ${ }^{6}$, Ikuo Fujii' \& Shun'ichi Kuroda ${ }^{1,2}$
}

'Graduate School of Bioagricultural Sciences, Nagoya University, Furo-cho, Chikusa-ku, Nagoya, Aichi 464-8601, Japan, ${ }^{2}$ The Institute of Scientific and Industrial Research, Osaka University, Mihogaoka, Ibaraki, Osaka 567-0047, Japan, ${ }^{3}$ Furukawa Electric Co., Ltd., Yawata-kaigandori, Ichihara, Chiba 290-8555, Japan, ${ }^{4}$ STARLITE Co., Ltd., Kamitoyama, Ritto, Shiga 520-3004, Japan, ${ }^{5}$ Functional Genomics Unit, RIKEN Center for Developmental Biology, Minatojima-minamimachi, Chuo-ku, Kobe, Hyogo 650-0047, Japan, ${ }^{6}$ Graduate School of Science and Technology, Kobe University, Rokkodai-cho, Nada-ku, Kobe, Hyogo 657-8501, Japan, ${ }^{7}$ Graduate School of Science, Osaka Prefecture University, Gakuen-cho, Naka-ku, Sakai, Osaka 599-8570, Japan.

When establishing the most appropriate cells from the huge numbers of a cell library for practical use of cells in regenerative medicine and production of various biopharmaceuticals, cell heterogeneity often found in an isogenic cell population limits the refinement of clonal cell culture. Here, we demonstrated high-throughput screening of the most suitable cells in a cell library by an automated undisruptive single-cell analysis and isolation system, followed by expansion of isolated single cells. This system enabled establishment of the most suitable cells, such as embryonic stem cells with the highest expression of the pluripotency marker Rex1 and hybridomas with the highest antibody secretion, which could not be achieved by conventional high-throughput cell screening systems (e.g., a fluorescence-activated cell sorter). This single cell-based breeding system may be a powerful tool to analyze stochastic fluctuations and delineate their molecular mechanisms.

\footnotetext{
ell-based technology is sustained by a wide variety of cell species, such as bacteria, yeast, insect and plant, as well as animal and human cells, for research and industrial use. In particular, cells utilized in regenerative medicine and producing various biopharmaceuticals, such as cytokines, antibodies, enzymes, proteins, peptides and metabolites, have significantly contributed toward human welfare. For the practical use of biopharmaceuticals, it is essential to obtain the most appropriate cells from a candidate cell population. Thus far, conventional cell screening systems have been based on colony isolation by assuming all cells in a colony possess homogeneous characteristics ${ }^{1,2}$. However, recent single cell-based analyses have revealed that each cell in an isogenic cell population shows diverse and heterogeneous gene expression, morphology and/ or cell proliferation ${ }^{3-5}$. For example, each cell of a mouse embryonic stem (ES) cell colony shows heterogeneous expression of the ES marker protein Rex $1^{6}$. Humanized immunoglobulin G (IgG)-producing Chinese hamster ovary $(\mathrm{CHO})$ cells are a mixture of clones showing stochastic fluctuations in IgG production ${ }^{7}$. Thus, a more rational approach has been considered necessary to isolate and culture the most suitable cells from a huge number of cell candidates by single-cell isolation and expansion (i.e., single cell-based breeding). Although we have examined the use of a fluorescence-activated cell sorter (FACS) as a practical approach, the recovered cells suffered from mechanical stresses probably associated with high voltage and pressure, as well as chemical stress from the sheath solution for cell suspension. A conventional FACS system requires cell sample consisting of $\sim 1 \times$ $10^{5}$ cell population and containing more than $0.1 \%$ positive cells ${ }^{8}$. Considering the cell sample may be limited, a large portion of the sample is required for FACS optimization, in which cell sample is not recovered. These current issues of FACS technology prompted us to develop a novel system capable of isolating single positive cells from less than $1 \times 10^{5}$ cells under undisruptive conditions. In this study, we have developed an automated singlecell analysis and isolation system to facilitate high-throughput isolation of fluorescently labeled mammalian cells in an undisruptive and single cell-based manner. The robot is a stand-alone machine with a microchamber array chip (containing $\sim 2.5 \times 10^{5}$ cells) and an automated micromanipulator with a glass capillary and fluorescence microscope system, which isolates single positive cells from $\sim 2.5 \times 10^{5}$ cells under undisruptive conditions. Here, we report single cell-based selection and expansion of mouse ES cells with a homogeneous genetic background for
} 
the pluripotency marker gene Rexl and hybridomas that highly secrete antibody, using our automated single-cell isolation system.

\section{Results}

Development of the automated single-cell analysis and isolation system. Recently, a microchamber array chip was developed, which allows single-cell microarray analysis of a large cell population $(\sim 2.0$ $\times 10^{5}$ cells $)^{8}$. Each microchamber $(10 \mu \mathrm{m}$ in diameter $)$ is designed to accommodate only one cell and enables weak fluorescence detection at a high signal to noise ratio by excluding noise signals from negative cells. However, both the fluorescence analysis and single-cell isolation of on-chip cells have been carried out manually. A fully automated single-cell isolation system may improve screening efficiency for the most appropriate cell from a candidate cell population. Therefore, we constructed a robot that executed the following procedures automatically. First, acquisition of the fluorescent intensity of each cell on the microchamber array chip. Second, permutation of cells by the order of their fluorescent intensities. Third, physical retrieval of desired cells with a glass capillary attached to a micromanipulator. Fourth, movement and release of isolated cells to the

a

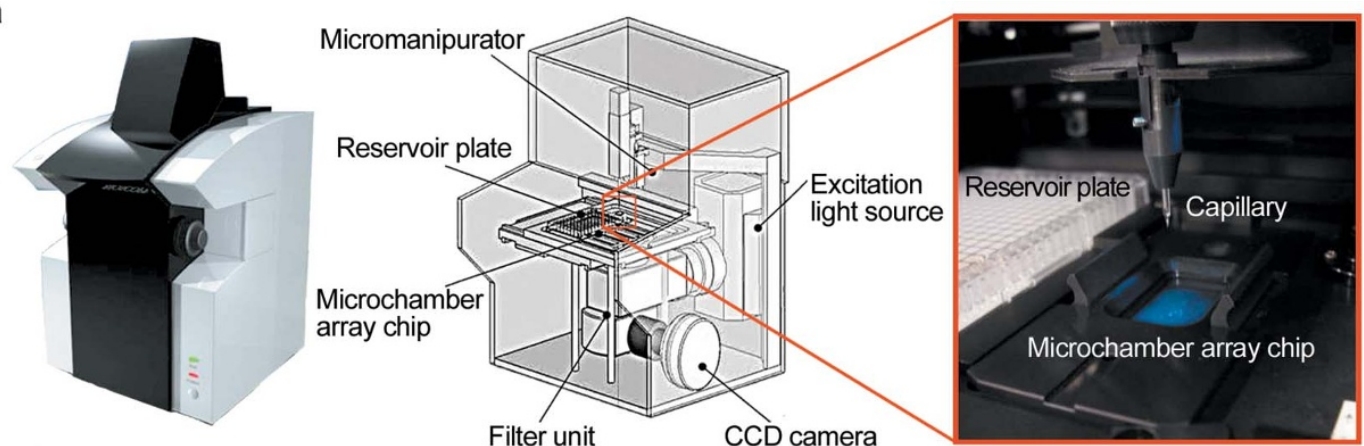

b

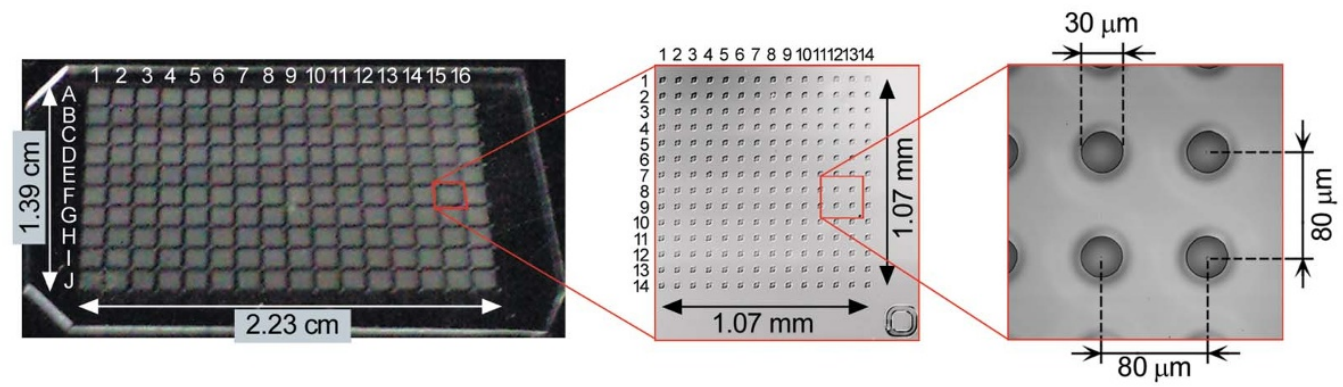

C

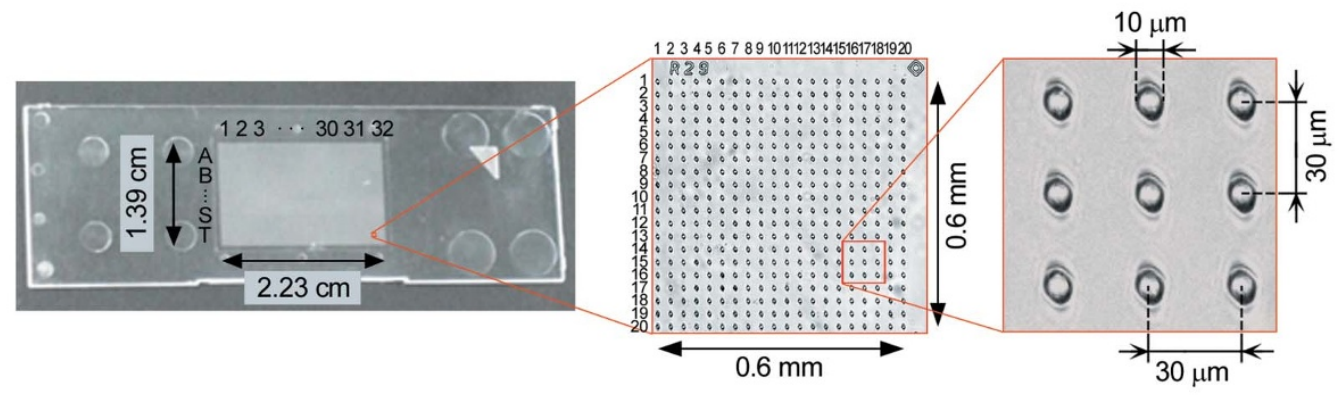

d

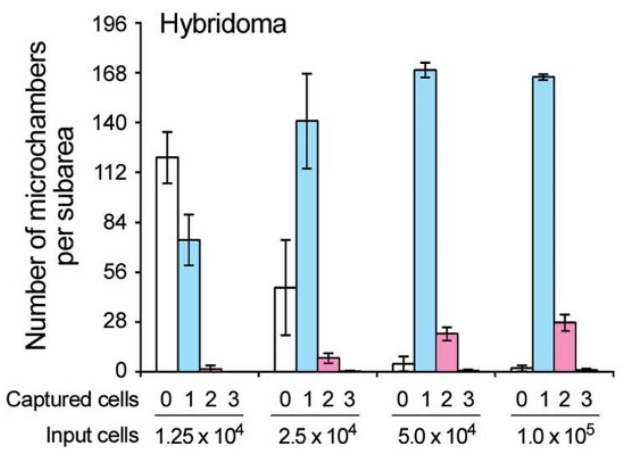

e

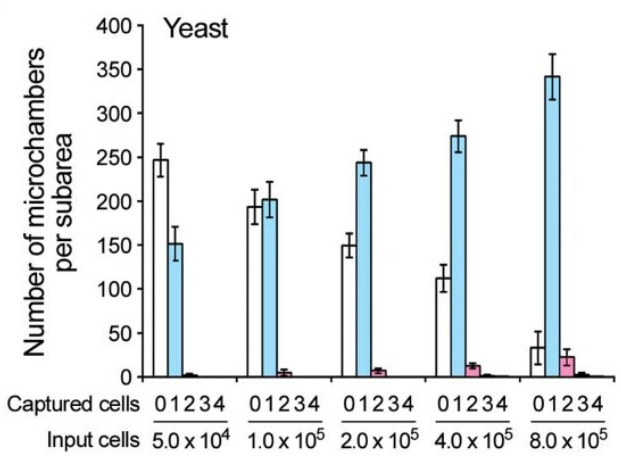

Figure 1 Overview of the automated single-cell analysis and isolation system. (a) Automated single-cell analysis and isolation system.

(b) Microchamber array chip made of polydimethylsiloxane (PDMS, 31,360 wells, $30 \mu \mathrm{m}$ diameters). Left, overview (10 $\times 16$ subareas); center, subarea $(14 \times 14$ wells); right, wells. (c) Microchamber array chip made of polystyrene (PS, 256,000 wells, $10 \mu \mathrm{m}$ diameters). Left, overview ( $20 \times 32$ subareas); center, subarea $(20 \times 20$ wells); right, wells. (d) Cell numbers of hybridoma in each 30- $\mu \mathrm{m}$ PDMS microchamber. Error bars $=$ SD $(\mathrm{n}=6)$.

(e) Cell numbers of yeast in each 10- $\mu \mathrm{m}$ PS microchamber. Cell numbers of hybridoma in each 30- $\mu \mathrm{m}$ PDMS microchamber. Error bars $=$ SD $(\mathrm{n}=6)$. 
a

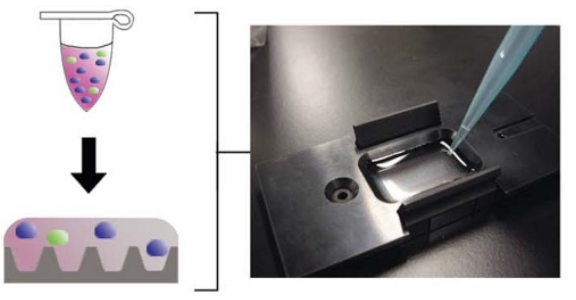

b

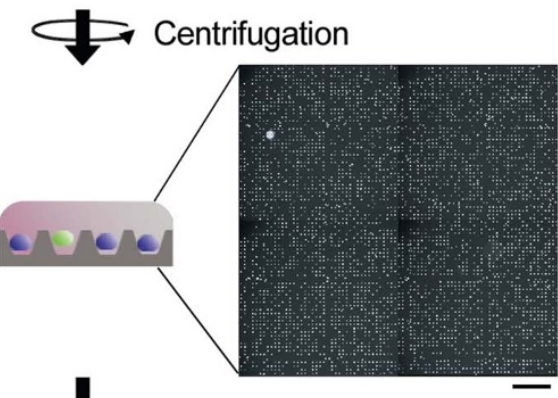

C
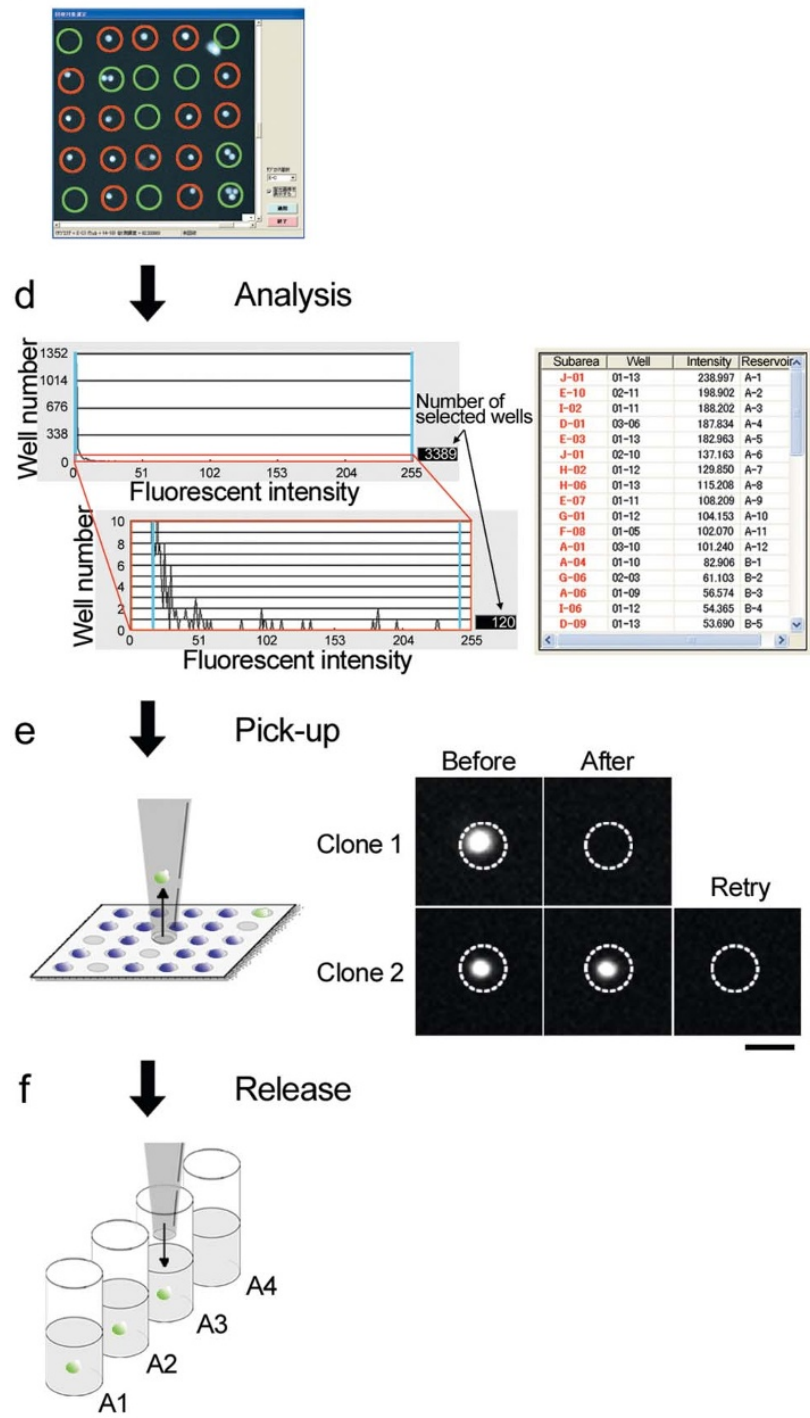

Figure 2 Flow chart of the automated single-cell analysis and isolation system. Approximately $5.0 \times 10^{4}$ cells in culture medium were added to the microchamber array chip equipped with an aluminum frame (step (a)) and then introduced into 30- $\mu \mathrm{m}$ PDMS microchambers by brief centrifugation $(50 \times g$, at room temperature for $1 \mathrm{~min})(\mathrm{step}(\mathrm{b}))$. The microchamber array was scanned with a CCD camera on the robot. Based on the fluorescent image, microchambers containing no or more than 2 fluorescent particles were excluded from further analyses. Green circles, excluded microchambers; red circles, selected microchambers (step (c)). A histogram of fluorescent intensity from each cell was generated for permutation of cells by the order of fluorescent intensities, a list of addresses, fluorescent intensities and images, as well as a transmission image of each cell was generated (step (d)). Cells of interest were automatically retrieved with a glass capillary attached to the micromanipulator. Recovery of each cell was repeated until the fluorescence from each microchamber was absent ( step (e)). Each retrieved cell was transferred to an assigned reservoir well (step (f)).

Scale bars $=200 \mu \mathrm{m}(\mathrm{b})$ and $30 \mu \mathrm{m}(\mathrm{e})$.

reservoirs of a multi-well plate (Figure 1a). Detailed specifications of the robot were described in "Methods section." Two types of microchamber array chips were fabricated with polydimethylsiloxane (PDMS) for mammalian cells $\left(31,360\right.$ wells on a $1.39 \times 2.23 \mathrm{~cm}^{2}$ total area; $30 \mu \mathrm{m}$ diameter, $80 \mu \mathrm{m}$ well-to-well pitch and $30 \mu \mathrm{m}$ well depth) (Figure 1b), and with polystyrene (PS) for small mammalian or yeast cells $\left(256,000\right.$ wells on a $1.39 \times 2.23 \mathrm{~cm}^{2}$ total area; $10 \mu \mathrm{m}$ diameter, $30 \mu \mathrm{m}$ well-to-well pitch and $10 \mu \mathrm{m}$ well depth) (Figure 1c). Various numbers of hybridomas were introduced into $30-\mu \mathrm{m}$ microchambers by brief centrifugation. Approximately $80 \sim 90 \%$ of microchambers were occupied by single hybridomas (Figure 1d). Similarly, yeast cells were introduced into 10- $\mu \mathrm{m}$ microchambers at comparable efficiency (Figure 1e).

Flowchart of the automated single-cell analysis and isolation system. Cell manipulation by the robot was carried out as follows. Cells were introduced into microchambers by brief centrifugation (Figure 2, steps a and b) and covered with culture medium, which could be cultured for at least $24 \mathrm{~h}$. The fluorescent intensities of 9,600 microchambers on a chip were measured by the robot for $30 \mathrm{~s}$ (14 min for a 256,000 microchamber array chip) (step b; Supplementary video S1 online). Microchambers containing no or more than 2 fluorescent particles were excluded from further analyses (step c). Finally, a histogram together with a list of correlations between the position and fluorescent intensity of each cell was generated (step d). Cells of interest could be virtually marked in a descending/ ascending/random order of fluorescent intensity. Marked cells were automatically collected with a glass capillary attached to the micromanipulator of the robot, which were confirmed by elimination of fluorescence in the target microchamber (step e). Upon failure, the robot automatically repeated the collection process. Each cell was transferred and released into the culture medium of an assigned well in 96- or 384-well plates (step f). The reciprocal movement of the glass capillary required $15 \mathrm{~s}$ for each cell (Supplementary video S2 online).

Single cell-based breeding of mouse ES cells. Among established ES cell lines, the expression of pluripotency markers in each cell has often been observed in a stochastic fluctuating state ${ }^{3,6}$. When $\sim 5.0$ $\times 10^{4}$ cells of the mouse ES cell line OLG harboring the Oct4-EGFP gene were introduced to 30- $\mu \mathrm{m}$ PDMS microchambers in our system, the cells showed variety of expression level of Oct4 (Figure 3a, upper panel). The mouse ES cell line clone No. 10 harboring the Rex1-EGFP gene showed an even higher degree of variety of expression level of Rex1 (Figure 3a, lower panel), indicating that each mouse ES cell line showed a distinct distribution of stemness?. From the cell library of clone No. 10 mouse ES cells, 24 cells with the highest fluorescent intensity were transferred to culture medium and allowed to proliferate from 1 to $\sim 1,000$ cells over $7 \mathrm{~d}$ (Figure $3 \mathrm{~b}$ ). The daughter cells formed rounder colonies with increased homogeneous Rex1-EGFP expression, compared with that of parental cells. After 2-3 weeks, 23 clones reached $\sim 1 \times 10^{6}$ cells, in which 
a

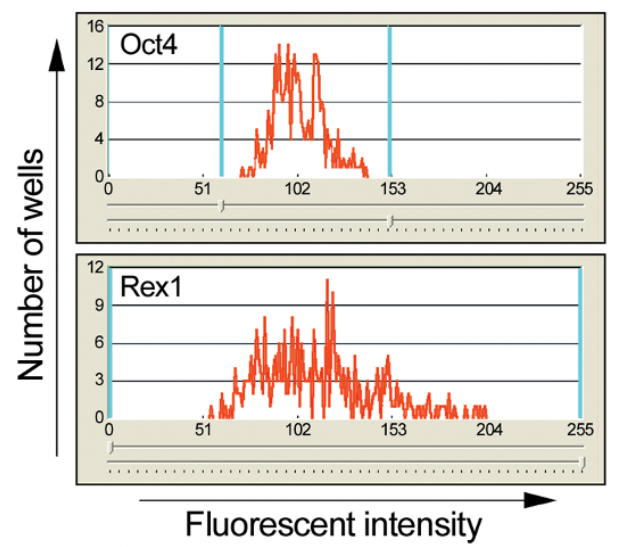

b

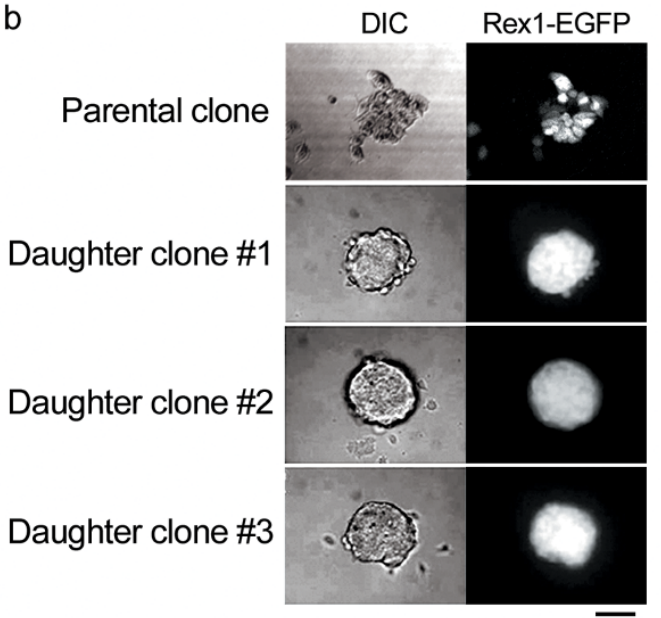

C

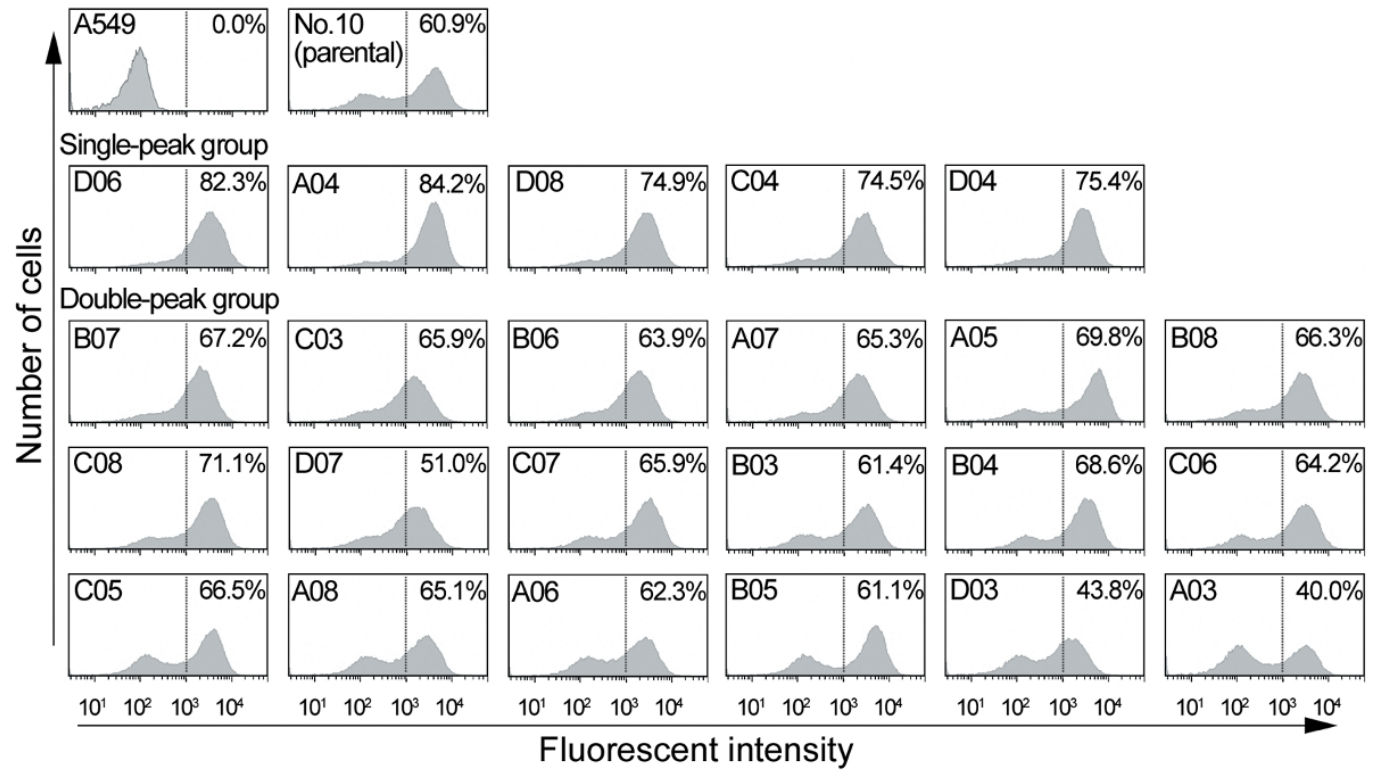

Figure 3 Single cell-based breeding of mouse ES cells. (a) Oct4-EGFP and Rex1-EGFP expression in mouse ES cell lines OLG and No. 10, respectively, were analyzed by the robot. (b) Colony formation from isolated No. 10 cells (daughter cells). Scale bar $=50 \mu \mathrm{m}$. (c) Rex1-EGFP expression of isolated No. 10 cells. Approximately $2.0 \times 10^{4}$ cells were analyzed by FACS. Clone numbers are indicated in the upper-left. Contents of cells with higher fluorescent intensity (over 10 $0^{3}$ ) are indicated in the upper-right. A549 (an adenosquamous lung carcinoma cell line) cells were used as a negative control.

20 clones retained a higher fluorescent intensity compared with that of the parental cell population (Figure $3 \mathrm{c}$ ). When calculating the ratio of highest numbers of cells with higher intensity (over $10^{3}$ ) to those with lower intensity $\left(10^{2} \sim 2 \times 10^{2}\right)$, the daughter cells of $>7.0$ ratio (mean +3 SD of parental cells, $n=6$ ) were judged as a single-peak group. Finally, we obtained 5 clones expressing higher level of Rex1, which would be suitable for further breeding process (Figure $3 \mathrm{c}$ ). This result indicated that single cell-based breeding of cells isolated from a cell library is a powerful method to expand ES cells with the highest expression of pluripotency markers. ES and induced pluripotent stem (iPS) cells, particularly from humans, are often susceptible to mechanical and chemical stresses ${ }^{10}$. The automated single-cell isolation system is practical for isolating suitable cells under undisruptive conditions because of gentle manipulation of cells in culture medium with a glass capillary.

Single cell-based breeding of hybridomas. To evaluate the secretion of anti-rabbit lactate dehydrogenase $\operatorname{IgG}_{1}$ from the hybridoma cell line HyLDH/YK-1, we treated the hybridomas with brefeldin A (a protein transport inhibitor $)^{11}$ to accumulate the $\operatorname{IgG}$ intracellularly, followed by FACS analysis. The isogenic cells were found to secrete various amounts of antibody and showed a dynamic fluorescent intensity range between $\sim 10^{2}$ and $\sim 10^{5}$ (Figure $4 \mathrm{a}$ ). A traditional limiting dilution method to isolate mammalian cells secreting high amounts of biopharmaceuticals, deposits the cell library into 96- or 384-well plates at $\sim 1$ cell per well to form colonies. After culturing for at least 1 month, the supernatants of these cell cultures are assayed by enzyme-linked immunosorbent assay (ELISA) to detect candidate clones, followed by establishing the selected cells, which is time consuming with low scalability (up to $\sim 100$ candidate clones per screen). Recently, two automatic single-colony isolation systems became commercially available (ClonePix FL (Molecular Devices, Sunnyvale, CA, USA) and CellCelector (AVISO, Jena, Germany)). Both machines facilitate manipulation of a large number of cells, but approximately 1 month is still required for colony formation and identification of candidate cells ${ }^{12,13}$. Using a cuboid microchamber array as the single-cell container and the solid phase of the ELISA, candidate cells can be manually detected after culturing cells for 
a

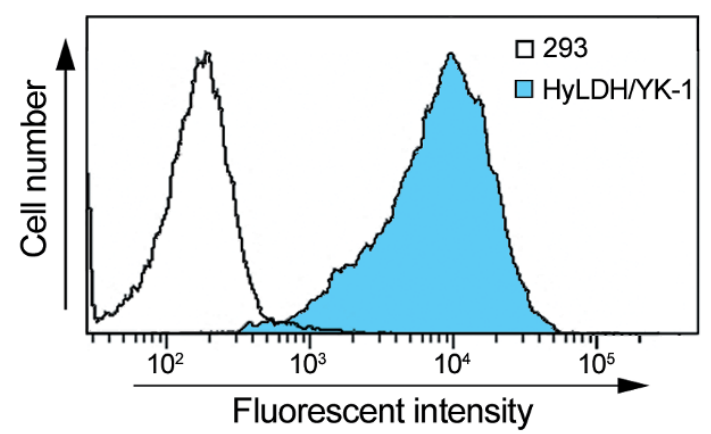

b

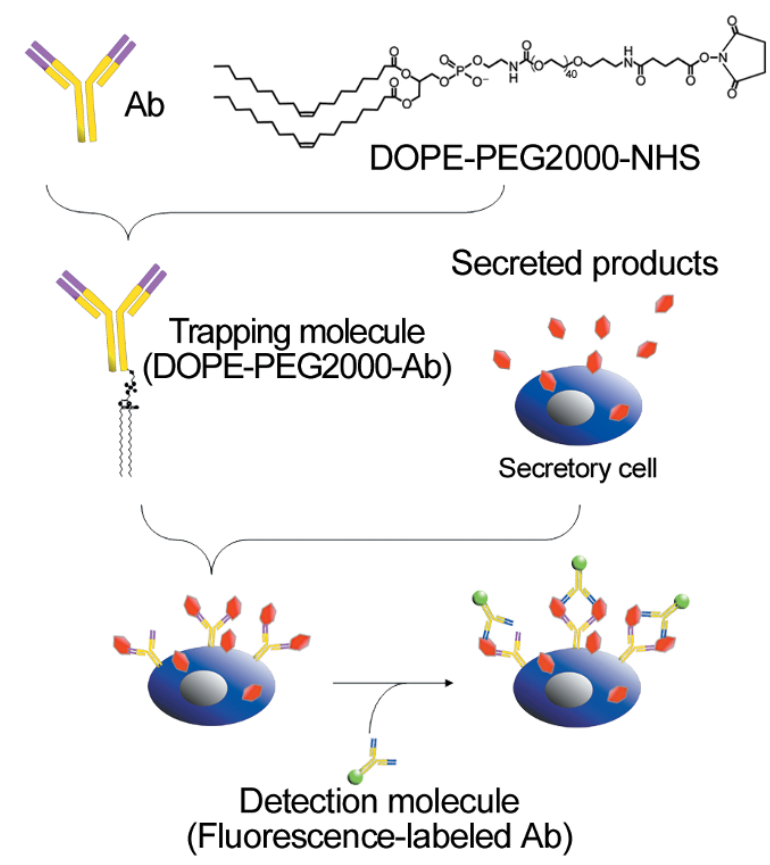

f

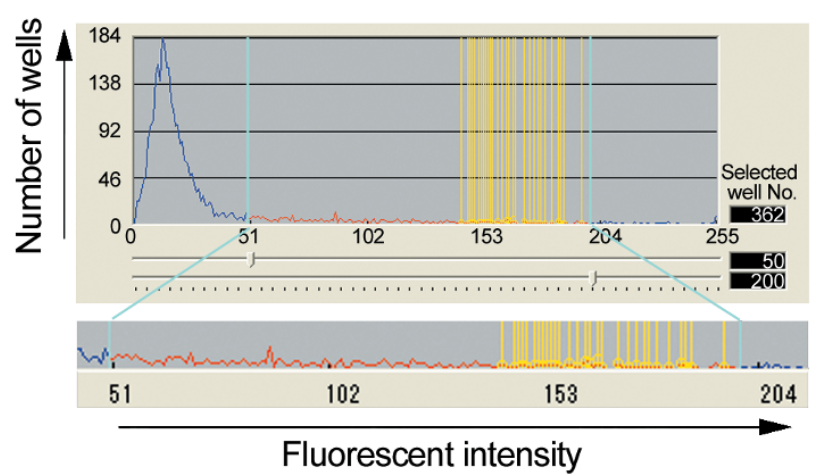

C

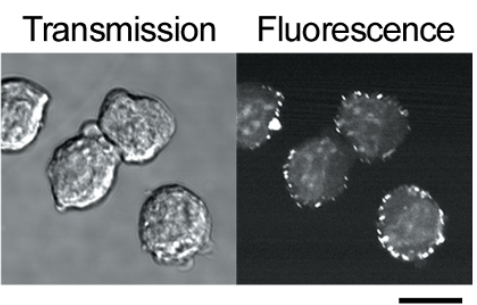

d

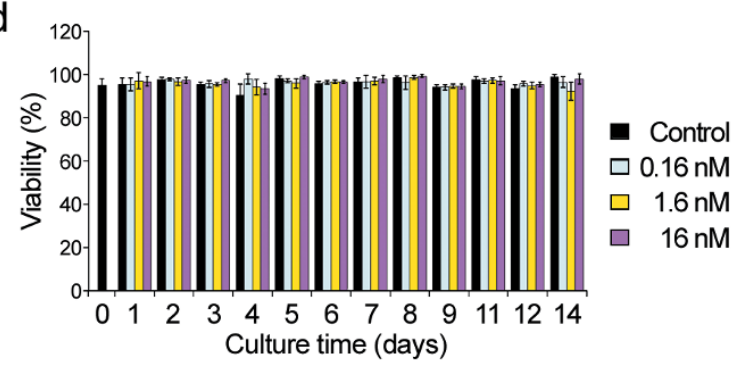

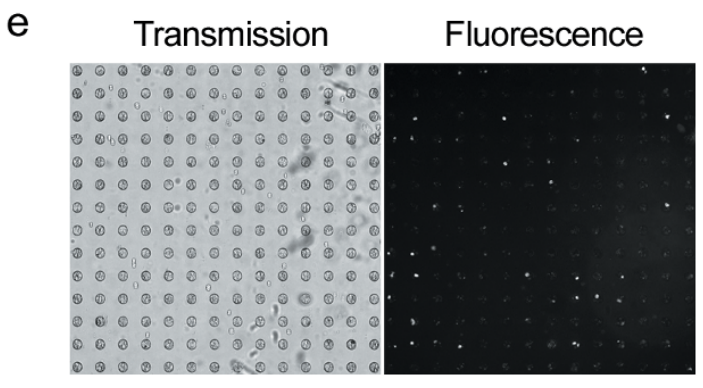

$\mathrm{g}$

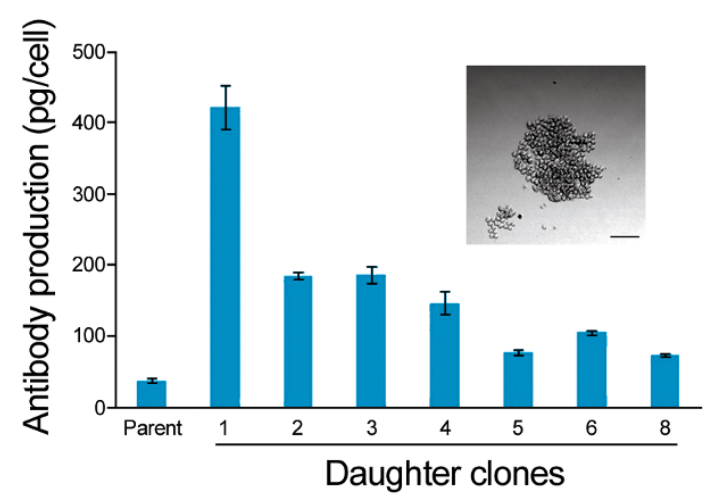

Figure $4 \mid$ Single cell-based breeding of hybridomas. (a) Antibody production by mouse hybridoma HyLDH/YK-1 cells was analyzed by FACS. HEK293 cells were used as a negative control. (b) Outline of the cell-surface FIA. Cells displaying trapping molecules, goat anti-mouse Fc antibody conjugated to DOPE-PEG2000, were allowed to capture secreted antibodies and then treated with a FITC-labeled rabbit anti-mouse $\mathrm{F}\left(\mathrm{ab}^{\prime}\right)_{2}$ antibody for detection. (c) Alexa 488-labeled trapping molecules on the surface of HyLDH/YK-1 cells. Scale bar $=20 \mu \mathrm{m}$. (d) Cytotoxicity of the trapping molecule on HyLDH/YK-1 cells. Cell viabilities were measured by trypan blue staining; error bars, $p<0.01, \mathrm{n}=12$ from three independent experiments.

(e) On-chip cell-surface FIA visualized by the robot. Scale bar $=240 \mu \mathrm{m}$. (f) Histogram of antibody secretion determined by the cell-surface FIA. Black vertical lines indicate fluorescence thresholds (range 50-200). White vertical lines indicate cells with high fluorescent intensities. (g) Antibody secretion determined by a conventional ELISA. Seven cells formed colonies at $\sim 10 \mathrm{~d}$ after single-cell isolation (inset, scale bar $=100 \mu \mathrm{m}$ ) and were subjected to ELISA at $\sim 20$ d. Error bars, $p<0.05, \mathrm{n}=6$ from three independent experiments. 
several days. The selected cells are then automatically isolated by CellCelector ${ }^{13}$. However, both machines are unable to promptly and automatically isolate the most suitable cell from a cell library, and retain the issue of cell heterogeneity in the isogenic cell population. Meanwhile, an automated single cell isolation system (Cellporter system; SC World, Toyama, Japan $)^{14}$ has been developed, which is likely to utilize similar picking-up system to our study. But, the machine requires additional scanning system (SC Scanner) for measurement of fluorescent intensity of each cell, indicating that Cellporter system is not a completely automated system for cell analysis and isolation. To identify hybridomas that secreted higher amounts of antibodies, we developed an undisruptive cell-surface fluorescence-linked immunosorbent assay (CS-FIA) to evaluate antibody production at a single-cell level using the robot (Figure $4 \mathrm{~b}$ ). The surfaces of secreting cells were modified with a goat anti-mouse Fc antibody conjugated to dioleoyl phosphatidylethanolamine-poly-ethylene glycol 2000 (DOPE-PEG2000) as a trapping molecule ${ }^{15,16}$. Trapping molecules spontaneously assembled around the cell surface (Figure 4c) and showed no cytotoxicity for at least 2 weeks (Figure 4d). Cells were introduced into 30- $\mu \mathrm{m}$ PDMS microchambers to allow the capture of secreted antibodies at the cell surface, followed by incubation with a fluorescein isothiocyanate (FITC)-labeled rabbit anti-mouse $\mathrm{F}\left(\mathrm{ab}^{\prime}\right)_{2} \mathrm{~F}\left(\mathrm{ab}^{\prime}\right)_{2}$ to establish the sandwich FIA on the cell surface (Figure 4e). After analyzing on-chip cells using the robot, 362 cells exhibited higher fluorescence among the $\sim 5.0 \times 10^{4}$ cells (Figure $4 \mathrm{f}$ ). Eight cells with the highest level of fluorescence were isolated. Seven cells formed colonies within $10 \mathrm{~d}$ and expanded to $\sim 5 \times 10^{5}$ cells over 2 weeks (Figure $4 \mathrm{~g}$ ). When evaluating the amount of antibody secreted into the culture medium by ELISA, the daughter cells were found to secrete higher amounts of antibodies, compared with that of the parental clone. This result indicated that single cell-based analysis and isolation allowed us to obtain hybridomas with the highest antibody-secreting ability in only 1 day. Because the CS-FIA technique is applicable to other cell types and various secreted biomaterials, the combination of the automated single-cell isolation system with the CS-FIA would be more effective to improve the productivity of biomaterials secreted from various cells.

Single cell isolation of adhesive cells. Since ES cells and hybridomas are less adhesive and non-adhesive cells, respectively, we herein examined if the robot is applicable to adhesive cells, such as $\mathrm{CHO}$ cells. CHO cells $\left(\sim 1.0 \times 10^{4}\right.$ cells $)$ were trypsinized, stained with CytoRed (fluorescent dye for viable cells; Dojin, Kumamoto, Japan) and then introduced into $30-\mu \mathrm{m}$ PDMS microchambers at comparable efficiency of ES cells and hybridomas (Figure 5a). Within $3 \mathrm{~h}$, single $\mathrm{CHO}$ cells could be retrieved according to their fluorescent intensities by the robot (Figure $5 \mathrm{~b}$ ), and proliferate to $\sim 4$ a

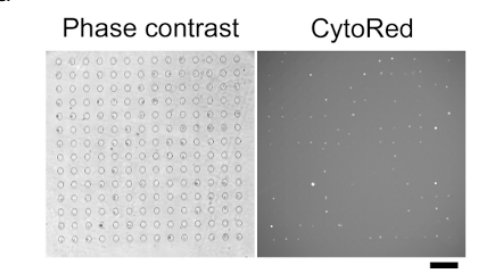

C

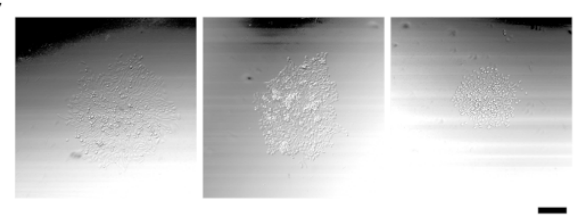

b

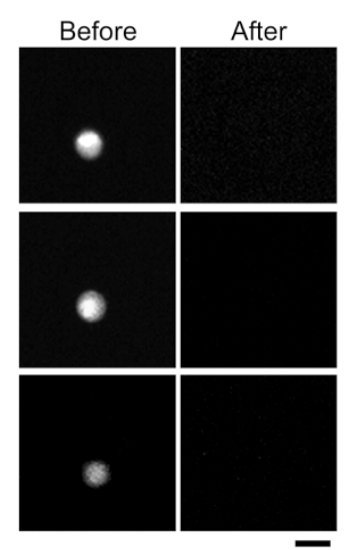

d

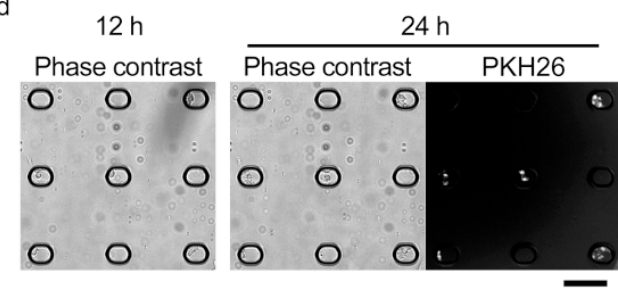

$f$
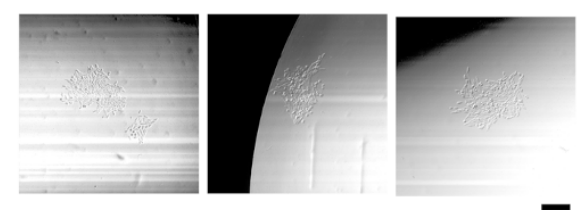

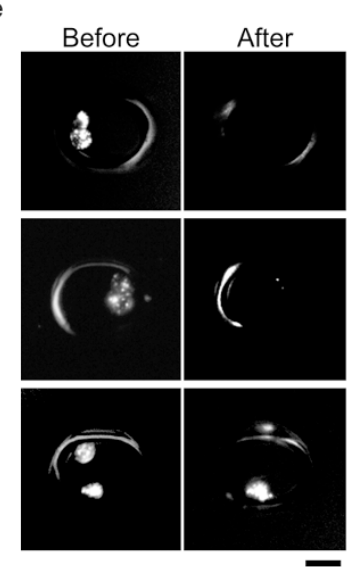

Figure 5 | Single cell isolation of CHO cells. (a) Cell array for CHO cells. Cells were stained with CytoRed solution. Scale bar $=160 \mu \mathrm{m}$. (b) Retrieval of single CHO cell. Scale bar $=25 \mu \mathrm{m}$. (c) Colony formation from isolated CHO cells. Scale bar $=200 \mu \mathrm{m}$. (d) On-chip culture of CHO cells. Cells were cultured on microchamber array chip and stained with PKH26. Scale bar $=100 \mu \mathrm{m}$. (e) Retrieval of cell cluster of CHO cells. CHO cells stained with PKH26 were trypsinized and retrieved with the robot. Scale bar $=25 \mu \mathrm{m}$. (f) Colony formation from isolated cell cluster of CHO cells. Scale bar $=$ $200 \mu \mathrm{m}$. 
$\times 10^{2}$ cells in 6 days (Figure $5 \mathrm{c}$ ), of which the viability was more than $80 \%$ of retrieved single cells (20/24 clones). Furthermore, single CHO cells transferred into $50-\mu \mathrm{m}$ ellipsoidal PS microchambers could propagate to several cells in microchambers (Figure 5d). After $24 \mathrm{~h}$, the cells were stained with PKH26 (fluorescent dye for membrane staining; Sigma-Aldrich, St Louis, MO, USA), trypsinized briefly, and then retrieved as a cell cluster by the robot (Figure 5e). These cell clusters were allowed to grow up to $\sim 2 \times 10^{2}$ cells in 5 days (Figure $5 \mathrm{f}$ ), of which the viability was nearly $100 \%$ of retrieved cell clusters (10/10 cell clusters). The automated retrieval of cell clusters might be useful for the single cell-based breeding of vulnerable adhesive cells.

\section{Discussion}

To manufacture biopharmaceuticals, each cell used for production (e.g., hybridomas and $\mathrm{CHO}$ cells) should express biomaterials stably and efficiently. In regenerative therapies, each stem cell should express pluripotency markers homogeneously to obtain differentiated cells with less tumorigenicity. It is an important step to isolate the most suitable cell from a candidate cell library and expand the cell population for further optimization. However, both isolation and expansion have been traditionally performed with a cell colony, which impairs isolation of suitable cells because of potential difficulties in excluding contaminating unsuitable cells. Furthermore, longterm culture necessary for colony formation often impairs the favorable properties of isolated cells ${ }^{7}$. For example, the productivity of established hybridomas is sometimes decreased by long-term cell culture $^{17}$. In established ES, iPS and mesenchymal stem cells, a trace number of cells in colonies cannot fully differentiate, which sometimes leads to severe tumorigenesis ${ }^{18,19}$. These cellular instabilities are mainly caused by stochastic fluctuation of gene expression among individual cells $\mathrm{s}^{3-7}$, which is considered a general phenomenon in various cell types. Although cellular instabilities have been conventionally circumvented by contingency-based cell screenings (i.e., random screening of naturally occurring suitable cells) to establish cells with low stochastic fluctuation, the molecular mechanisms underlying stochastic fluctuation should be delineated. For example, after purifying two types of cells with high and low stochastic fluctuation by single-cell isolation, a comparison of gene expression profiles might delineate the mechanisms. Taken together, cells used for producing biopharmaceuticals and regenerative therapies should be established by single cell-based screening to maximize favorable properties and minimize the occurrence of stochastic fluctuation.

Here, we demonstrated that an automated single-cell isolation system facilitates prompt and effective establishment of the most suitable single cells from a large number of candidate cells to avoid the occurrence of stochastic fluctuation. Mouse ES cells with the highest expression level of the pluripotency marker Rex1 were successfully isolated from a heterogeneous cell population. Although 3 isolated ES cells (clones D07, A03, D03) showed lower expression level of Rex1 at 2-3 weeks after single-cell isolation, 20 cells retained higher Rex1 expression (see Figure 3c). Particularly, 5 isolated ES cells (clones D06, A04, D08, C04, D04) were found to show homogeneous profile of Rex1 expression. In addition, hybridomas secreting the highest amount of antibodies were isolated by the robot in combination with a CS-FIA technique. All isolated hybridomas showed a higher expression level of antibody than that of parental hybridomas at $\sim 21$ days after single-cell isolation (see Figure $4 \mathrm{~g}$ ). These results indicate that single cell-based breeding is a practical way to obtain suitable cells with fewer effects from stochastic fluctuation. Moreover, these established cells would be useful to decipher the molecular basis of stochastic fluctuation.

Micromanipulation under a microscope, limiting dilution and FACS have been widely used to isolate single cells. Micromanipulation is undisruptive and reliable for manipulating single cells, but extremely laborious. Limiting dilution is also undisruptive, but not reliable because of possibly excluding suitable cells during serial dilutions. As described in the introduction, FACS is a high-throughput and reliable system for isolating cell populations, but cannot isolate a single positive cell from less than $1 \times 10^{5}$ cells under undisruptive conditions. In particular, the chemical and mechanical stresses intrinsically associated with FACS are likely to alter the gene expression profiles of isolated single cells, thus single-cell manipulation should be carried out under undisruptive conditions. The issues of these techniques for isolating single cells have led us to develop an automated single-cell isolation system. The robot is designed to isolate a single positive cell from less than $1 \times 10^{5}$ cells in about $1 \mathrm{ml}$ of culture medium. Therefore, the automated single-cell isolation system is considered suitable for high-throughput single cell-based analysis and isolation.

In high-throughput identification of hybridomas that secrete high amounts of antibodies, candidate hybridomas can be selected by FACS, followed by colony formation and evaluation with a conventional ELISA. Cell-surface markers (e.g., CD19, CD20, CD38, CD138 $)^{20-24}$ expressed in hybridomas are indirectly labeled with fluorescence, and then populations of positive cells are collected as candidate hybridomas by FACS. This method cannot evaluate the antibody-secreting ability of each hybridoma directly, and only concentrates candidate hybridomas. Another method is treatment of cells with brefeldin A to accumulate antibodies intracellularly. This method can evaluate the antibody-secreting ability, but also severely affects cell viability. Thus far, no reported method can evaluate the antibody-secreting ability of hybridomas directly in an undisruptive manner and without the requirement of long-term culture for colony formation. Here, we developed a CS-FIA that fulfills the above criteria. Combination of the CS-FIA with the robot successfully shortened the time to identify positive hybridomas from $\sim 1$ month (FACS) or $\sim 2$ months (limiting dilution) to 1 day. To further optimize the CS-FIA, the use of fluorescence-labeled antigens as detection molecules could improve the signal to noise ratio by reducing cross reactions among displayed antibodies.

In addition to single cell-based high-throughput analysis and isolation, our robot can be used for the following applications. Because the robot can evaluate protein-protein interactions on the cell surface, orphan ligand screenings using cells displaying receptor libraries, and orphan receptor screenings using cells expressing cell surface-anchored forms of ligand libraries are applicable, which may lead to drug discovery. Moreover, because of the undisruptive condition during cell manipulation, the robot would be suitable for isolation of rare cells in limited clinical samples (e.g., circulating tumor cells ${ }^{25}$ and cancer stem cells ${ }^{26}$ ) to develop an innovative diagnosis system. Thus, our automated single-cell isolation system may offer a new technology for single cell-based engineering and bring significant progress to cell-based research and industries.

\section{Methods}

The automated single-cell analysis and isolation system. The robot consisted of a micromanipulator $(<1 \mu \mathrm{m}$ movement accuracy) equipped with a pencil pump (DENSO, Aichi, Japan) and glass capillary $(40.5 \mu \mathrm{m}$ inside diameter, $58 \mu \mathrm{m}$ outside diameter for mammalian cell; $14.5 \mu \mathrm{m}$ inside diameter, $21 \mu \mathrm{m}$ outside diameter for yeast cell), a CCD camera (Nikon, Tokyo, Japan), an objective lens (Plan Fluor $10 \times /$ 0.3 , Nikon), an excitation light source (Nikon) using a super high pressure mercury lamp and power supply, filter units (for FITC, 465-495-nm excitation, 515-555-nm emission, $505 \mathrm{~nm}$ dichroic mirror; for Cy3, 510-560-nm excitation, 572.5-647.5-nm emission, $565 \mathrm{~nm}$ dichroic mirror, Nikon), a 96- or 384-well reservoir plate, a microchamber array chip and a control PC (CPU, Pentium M 1.8 GHz; RAM $980 \mathrm{MB}$ ). Microfabrication on the polymethylmethacrylate (PMMA) plate was performed by X-ray mask and lithography technology combined with synchrotron radiation $^{8,27}$. Using the patterned PMMA plate as a master plate, a nickel $(\mathrm{Ni})$ mold part was produced by an electro-forming process resulting in effective production of the Ni mold part with replicated micro-patterns. Then, the mold part was inserted into a mold base and injected to produce a microchamber array chip with polystyrene or PDMS. The robot identified a minimum $1 \mu \mathrm{m}$ single particle at $<1800 \mathrm{MESF}$ from the fluorescence of GFP, EGFP, phycoerythrin and propidium iodide. The control PC regulated the vertical movement of the micromanipulator, the horizontal movement 
of the microchamber array chip, fluorescence acquisition and transmission of images to indentify cells of interest, followed by cell recovery using the pencil pump.

Cell culture. Mouse ES cell lines, OLG and clone No. 10, were cultured in Glasgow Minimum Essential Medium (Sigma-Aldrich, St Louis, MO, USA) supplemented with $10 \%$ fetal bovine serum (FBS; StemCell Technologies, Vancouver, BC, Canada), $1 \mathrm{mM}$ sodium pyruvate, $0.1 \mathrm{mM}$ non-essential amino acids (NEAA; Invitrogen Life Technology, Carlsbad, CA, USA), $32.7 \mathrm{mM}$ sodium bicarbonate, $0.1 \mathrm{mM} \mathrm{2-}$ mercaptoethanol and 1,000 $\mathrm{U} \mathrm{ml}^{-1}$ leukemia inhibitory factor (Invitrogen). The mouse hybridoma cell line HyLDH/YK-1 secreting anti-rabbit lactate dehydrogenase $\mathrm{IgG}_{1}$ was cultured in RPMI-1640 (Nacalai, Kyoto, Japan) supplemented with $10 \%$ FBS (Thermo, Waltham, MA, USA), $0.1 \mathrm{mM}$ NEAA and $0.05 \mathrm{mM} 2-$ mercaptoethanol. Human embryonic kidney (HEK) 293 and lung adenocarcinoma A549 cell lines were maintained in Dulbecco's modified Eagle's medium supplemented with 10\% FBS (PAA Laboratories, Pasching, Austria). Dihydrofolate reductase-deficient Chinese hamster ovary $(\mathrm{CHO})$ cells (CHO-DG44 cells) were maintained in RPMI-1640 supplemented with 10\% FBS (PAA Laboratories).

Flow cytometric analysis. Daughter mouse ES cells isolated by the robot were cultured for $2-3$ weeks to reach $\sim 1 \times 10^{6}$ cells, and then harvested by trypsinization. The fluorescent intensities of $\sim 2.0 \times 10^{4}$ cells from each clone were measured with a BD FACSCanto II flow cytometer (BD, Franklin Lakes, NJ, USA). Parental mouse ES and A549 cells were used as positive and negative controls, respectively. HyLDH/YK1 cells treated with $1 \mu \mathrm{g} \mathrm{ml}^{-1}$ brefeldin A (BD) for $6 \mathrm{~h}$ were fixed in PBS containing $4 \%$ paraformaldehyde (PFA) at room temperature for $15 \mathrm{~min}$, permeabilized with PBS containing $0.03 \%$ Triton X-100 at room temperature for $5 \mathrm{~min}$ and then collected by centrifugation $(200 \times g, 5 \mathrm{~min})$. Cells were then incubated with $2 \mu \mathrm{g} \mathrm{ml}^{-1}$ FITC-conjugated anti-mouse $\mathrm{F}\left(\mathrm{ab}^{\prime}\right)_{2}$ rabbit $\mathrm{F}\left(\mathrm{ab}^{\prime}\right)_{2}$ (Rockland, Gilbertsville, PA, USA) in PBS at room temperature for $20 \mathrm{~min}$, followed by FACS analysis. HEK 293 cells were used as a negative control.

Preparation of trapping molecules. For the cell-surface FIA, $1.65 \mathrm{nM}$ Immunopure anti-mouse Fc goat polyclonal antibody (Thermo) was reacted with $66 \mu \mathrm{M}$ DOPEPEG2000-N-hydroxysuccinimide (NHS; NOF, Tokyo, Japan) in $100 \mu$ sodium phosphate $(10 \mathrm{mM}, \mathrm{pH} 7.6)$ and $250 \mathrm{mM} \mathrm{NaCl}$ at room temperature for $10 \mathrm{~min}$. Free DOPE-PEG2000-NHS molecules were removed by ultrafiltration through $50 \mathrm{kDa}$ cut-off membranes (Millipore, Billerica, MA, USA). Coupling of DOPE-PEG2000 to the antibody was confirmed by SDS-PAGE and Coomassie Brilliant Blue R-250 staining. To observe trapping molecules on the HyLHD/YK-1 cell surface, $\sim 2.0 \times 10^{4}$ cells were combined with $100 \mu \mathrm{l}$ serum-free RPMI-1640 medium containing $16 \mathrm{nM}$ trapping molecules, incubated at $37^{\circ} \mathrm{C}$ for $10 \mathrm{~min}$ and then fixed with $4 \% \mathrm{PFA}$ at room temperature for $20 \mathrm{~min}$. Cells were washed with PBS twice, incubated in $100 \mu \mathrm{l}$ PBS containing 400 ng Alexa 488-conjugated anti-goat IgG antibody (Invitrogen) at room temperature for $20 \mathrm{~min}$, washed with PBS twice and then observed under a confocal laser-scanning microscope (FV-1000D; Olympus, Tokyo, Japan). To evaluate the cytotoxicity of the trapping molecule (DOPE-PEG2000-antibody) in $\mathrm{HyLDH} / \mathrm{YK}-1$ cells, $\sim 1.25 \times 10^{5}$ cells in $2.5 \mathrm{ml}$ culture medium were treated with the trapping molecule at $0.16-16 \mathrm{nM}$ final concentration (as a protein concentration) and then maintained at $37^{\circ} \mathrm{C}$ with $5 \% \mathrm{CO}_{2}$ for 2 weeks. Cell viabilities were measured by trypan blue staining.

Cell-surface FIA. Approximately $5.0 \times 10^{4} \mathrm{HyLDH} / \mathrm{YK}-1$ cells were combined with $100 \mu \mathrm{l}$ serum-free RPMI-1640 medium containing $16 \mathrm{nM}$ trapping molecules, incubated at $37^{\circ} \mathrm{C}$ for $10 \mathrm{~min}$, mixed with $900 \mu \mathrm{l}$ serum-free RPMI-1640 medium and then placed into microchambers by brief centrifugation. After addition of $100 \mu \mathrm{l}$ FBS, cells on the microchamber array chip were incubated for $30 \mathrm{~min}$ in $\mathrm{a} \mathrm{CO}_{2}$ incubator to allow antibody secretion and then treated with $2 \mu \mathrm{g} \mathrm{ml}^{-1}$ FITC-labeled anti-mouse $\mathrm{F}\left(\mathrm{ab}^{\prime}\right)_{2} \mathrm{~F}\left(\mathrm{ab}^{\prime}\right)_{2}$ antibody to establish the cell-surface FIA. After incubation at room temperature for $10 \mathrm{~min}$, cells were briefly washed twice with medium and then subjected to the automated single-cell analysis and isolation system.

Mouse IgG ELISA. Each well of a MaxiSorp 96-well plate (Thermo) was treated with $30 \mu \mathrm{l}$ anti-mouse $\mathrm{Fc}$ antibody $\left(10 \mathrm{ng} \mu \mathrm{l}^{-1}\right)$ at $4{ }^{\circ} \mathrm{C}$ for $24 \mathrm{~h}$, washed with $200 \mu \mathrm{l}$ PBS containing $0.1 \%$ ( $\mathrm{vol} / \mathrm{vol}$ ) Tween 20 (PBS-T) five times and then treated with $200 \mu \mathrm{l}$ bovine serum albumin $\left(5 \mu \mathrm{g}^{-1}\right)$ in PBS at room temperature for $1 \mathrm{~h}$. To measure the amount of secreted antibody from $\mathrm{HyLDH} / \mathrm{YK}-1$ cells isolated by the robot, each sample was added to an anti-mouse Fc-immobilized well, incubated at room temperature for $1 \mathrm{~h}$ and then washed with $200 \mu \mathrm{l}$ PBS-T five times, followed by addition of $200 \mu \mathrm{l}$ PBS-T containing $0.2 \mu \mathrm{l}$ horse radish peroxidase-conjugated antimouse Ig (GE healthcare, Little Chalfont, Buckinghamshire). Plates were incubated at room temperature for $1 \mathrm{~h}$ and then washed with PBS-T five times, followed by addition of $100 \mu \mathrm{l} 3,3^{\prime}, 5,5^{\prime}$-tetramethylbenzidine substrate (Thermo). The coloration reaction was performed at room temperature for $30 \mathrm{~min}$, stopped by $100 \mu \mathrm{l} \mathrm{H}_{2} \mathrm{SO}_{4}$ $(2 \mathrm{M})$ and then the absorbance at $450 \mathrm{~nm}$ was measured.

1. Caron, A. W. et al. Fluorescent labeling in semi-solid medium for selection of mammalian cells secreting high-levels of recombinant proteins. BMC Biotechnol. 9, 42 (2009).

2. Huangfu, D. et al. Induction of pluripotent stem cells by defined factors is greatly improved by small-molecule compounds. Nat. Biotechnol. 26, 795-797 (2008).
3. Kurimoto, K., Yabuta, Y., Ohinata, Y. \& Saitou, M. Global single-cell cDNA amplification to provide a template for representative high-density oligonucleotide microarray analysis. Nat. Protoc. 2, 739-752 (2007).

4. Wang, D. \& Bodovitz, S. Single cell analysis: the new frontier in 'omics'. Trends Biotechnol. 28, 281-290 (2010).

5. Narsinh, K. H. et al. Single cell transcriptional profiling reveals heterogeneity of human induced pluripotent stem cells. J. Clin. Invest. 121, 1217-1221 (2011).

6. Toyooka, Y., Shimosato, D., Murakami, K., Takahashi, K. \& Niwa, H. Identification and characterization of subpopulations in undifferentiated ES cell culture. Development 135, 909-918 (2008).

7. Pilbrough, W., Munro, T. P. \& Gray, P. Intraclonal protein expression heterogeneity in recombinant CHO cells. PLoS One 4, e8432 (2009).

8. Yamamura, S. et al. Single-cell microarray for analyzing cellular response. Anal. Chem. 77, 8050-8056 (2005).

9. Casanova, J. Stemness as a cell default state. EMBO Rep. 13, 396-397 (2012)

10. Ohgushi, M. \& Sasai, Y. Lonely death dance of human pluripotent stem cells: ROCKing between metastable cell states. Trends Cell Biol. 21, 274-282 (2011).

11. Mendez, A. J. Monensin and brefeldin A inhibit high density lipoproteinmediated cholesterol efflux from cholesterol-enriched cells. Implications for intracellular cholesterol transport. J. Biol. Chem. 270, 5891-5900 (1995).

12. Serpieri, F. et al. Comparison of humanized IgG and FvFc anti-CD3 monoclonal antibodies expressed in CHO cells. Mol. Biotechnol. 45, 218-225 (2010).

13. Choi, J. H. et al. Development and optimization of a process for automated recovery of single cells identified by microengraving. Biotechnol. Prog. 26, 888-895 (2010)

14. Suzuki, M., Tanaka, H. \& Iribe, Y. Detection and Collection System of Target Single Cell Based on pH and Oxygen Sensing. J. Rob. Mechatron. 22, 639-643 (2010)

15. Kato, K. et al. Immobilized culture of nonadherent cells on an oleyl poly(ethylene glycol) ether-modified surface. Biotechniques 35, 1014-1018, 1020-1021 (2003).

16. Kato, K., Itoh, C., Yasukouchi, T. \& Nagamune, T. Rapid protein anchoring into the membranes of Mammalian cells using oleyl chain and poly(ethylene glycol) derivatives. Biotechnol. Prog. 20, 897-904 (2004).

17. Frame, K. K. \& Hu, W. S. The loss of antibody productivity in continuous culture of hybridoma cells. Biotechnol. Bioeng. 35, 469-476 (1990).

18. Ben-David, U. \& Benvenisty, N. The tumorigenicity of human embryonic and induced pluripotent stem cells. Nat. Rev. Cancer 11, 268-277 (2011).

19. Fong, C. Y., Peh, G. S., Gauthaman, K. \& Bongso, A. Separation of SSEA-4 and TRA-1-60 labelled undifferentiated human embryonic stem cells from a heterogeneous cell population using magnetic-activated cell sorting (MACS) and fluorescence-activated cell sorting (FACS). Stem Cell Rev. 5, 72-80 (2009).

20. Tedder, T. F. \& Isaacs, C. M. Isolation of cDNAs encoding the CD19 antigen of human and mouse B lymphocytes. A new member of the immunoglobulin superfamily. J. Immunol. 143, 712-717 (1989).

21. Chilosi, M. et al. CD138/syndecan-1: a useful immunohistochemical marker of normal and neoplastic plasma cells on routine trephine bone marrow biopsies. Mod. Pathol. 12, 1101-1106 (1999).

22. Harper, D. et al. In vitro and in vivo investigation of a novel monoclonal antibody to plasma cells (W5 mAb). Xenotransplantation 11, 78-90 (2004).

23. Polson, A. G. et al. Expression pattern of the human FcRH/IRTA receptors in normal tissue and in B-chronic lymphocytic leukemia. Int. Immunol. 18, 1363-1373 (2006).

24. Rosa, E. A., Lanza, S. R., Zanetti, C. R. \& Pinto, A. R. Immunophenotyping of classic murine myeloma cell lines used for monoclonal antibody production. Hybridoma (Larchmt) 31, 1-6 (2012).

25. Pantel, K. \& Alix-Panabières, C. Circulating tumour cells in cancer patients: challenges and perspectives. Trends Mol. Med. 16, 398-406 (2010).

26. Gupta, P. B. et al. Identification of selective inhibitors of cancer stem cells by highthroughput screening. Cell 138, 645-659 (2009).

27. Kurokawa, M. Advanced Micro \& Nanosystems, LIGA and Its Applications (Saile, V., Wallrabe, U., Tabata, O. \& Korvink, J. G. eds) 7, 323-335 (Wiley-VCH GmbH \& Co. KGaA, 2009).

\section{Acknowledgments}

This study was supported in part by The Japan Science Society (H22 to N.Y.), Adaptable \& Seamless Technology Transfer Program through Target-driven R\&D (A-STEP) by the Japan Science and Technology Agency (JST) (AS231Z04687F to N.Y., AS2311699F to S.K.), Regional New Consortium Projects (H15 to S.K. and H17 to I.F., METI, Japan), the Program for Promotion of Basic and Applied Researches for Innovations in Bio-oriented Industry (H22-7, BRAIN to S.K., K. Tanizawa), the Health Labor Sciences Research Grant from the Ministry of Health Labor and Welfare (to SK), a Grant-in-Aid for Scientific Research (A) (21240052 to S.K.), the As One Corporation (to S.K., A. Kondo, I.F.), the Suzuken Memorial Foundation (10-013 to S.K.), the Nagase Science and Technology Foundation (H22-1 to S.K.) and the Canon Foundation (H22-1-7 to S.K.). We thank Satoshi Sugiyama and Ken-ichi Kimura (Furukawa) for technical support, and Masahiro Matsushita and Kenji Uemukai (As One) for advice.

\section{Author contributions}

N.Y. and S.K. conceived the project, designed and performed experiments, processed and analyzed data and wrote the manuscript. A. Kida, M.I., T.N., A.D.M. and K. Tatematsu 
processed and analyzed data. X.J. and M.K. developed the robot and chips. I.N. and H.R.U. prepared mouse ES cell samples. A. Kondo, I.F., K. Tanizawa and S.K. coordinated the project.

\section{Additional information}

Supplementary information accompanies this paper at http://www.nature.com/ scientificreports
Competing financial interests: The authors declare no competing financial interests. License: This work is licensed under a Creative Commons

Attribution-NonCommercial-NoDerivs 3.0 Unported License. To view a copy of this license, visit http://creativecommons.org/licenses/by-nc-nd/3.0/

How to cite this article: Yoshimoto, N. et al. An automated system for high-throughput single cell-based breeding. Sci. Rep. 3, 1191; DOI:10.1038/srep01191 (2013). 